\title{
Trajectories of pain in patients undergoing lung cancer surgery: a longitudinal prospective study
}

Running title: Pain trajectories after lung cancer surgery

Kari Hanne Gjeilo, RN PhD, Trine Oksholm, RN, PhD, Turid Follestad, PhD, Alexander Wahba, MD $\mathrm{PhD}$, Tone Rustøen, RN PhD.

Department of Cardiothoracic Surgery (K.H.G., A.W.), Department of Cardiology (K.H.G.) and National Competence Centre for Complex Symptom Disorders (K.H.G.), St. Olavs Hospital, Trondheim University Hospital, Trondheim, Norway; Department of Circulation and Medical Imaging (K.H.G., A.W.) and Department of Public Health and Nursing (K.H.G., T.F.), Faculty of Medicine and Health Sciences, NTNU, Norwegian University of Science and Technology

Trondheim, Norway; VID Specialized University (T.O.), Haraldsplass, Bergen, Norway;

Department of Nursing Science (T.R.), Faculty of Medicine, University of Oslo, Oslo, Norway; Department of Research and Development (T.R.), Division of Emergencies and Critical Care, Oslo University Hospital, Ullevål, Oslo, Norway.

Tables: 3

Supplementary tables: 1

Figures: 6

Word count: 3568

References: 51 
Address correspondence to: Kari Hanne Gjeilo, Department of Cardiothoracic Surgery, St. Olavs Hospital, PO Box 3250 Torgarden, NO-7006 Trondheim, Norway. E-mail: kari.hanne.gjeilo@stolav.no 


\begin{abstract}
Context. Lung cancer surgery is among the surgical procedures associated with the highest prevalence of pain, but prospective longitudinal studies following the pain trajectory are scarce.
\end{abstract}

Objectives. We aimed to describe the pain trajectory in patients undergoing surgery for primary lung cancer and to investigate whether distinct groups of patients could be identified based on different pain trajectories.

Methods. Patients ( $n=264,95 \%$ thoracotomies) provided data on the average and worst pain intensity, pain location, and comorbidities before, and at 1, 5, 9, and 12 months after surgery. Pain profiles were analyzed by latent class mixed models (LCMMs).

Results. The occurrence of any pain increased from $40 \%$ before surgery to $69 \%$ after 1 month, and decreased to 56\%, 57\%, and 55\% at 5, 9, and 12 months, respectively. LCMMs identified two classes both for average and worst pain; one class started low with high ratings after one month, then returning to a level slightly higher than baseline. The other class started higher with similar scores through the trajectory. Patients reporting no pain (8\%) were placed in a separate class. Higher comorbidity score, preoperative use of pain and psychotropic medicine characterized the class with overall highest pain for average and/or worst pain.

Conclusion. Pain was highly prevalent after surgery and subgroups could be identified based on different pain trajectories. Patients reported both postoperative pain and pain from chronic conditions. Knowledge about vulnerable patients and risk factors for pain is important to tailor interventions and information about pain.

Key Words: Lung cancer, pain trajectory, chronic pain, persistent postsurgical pain, thoracic surgery. 
Key Message: This article describes a prospective study where pain was highly prevalent during the first year after surgery for lung cancer, and subgroups could be identified based on different pain trajectories. Patients reported both postoperative pain and pain from chronic conditions. This knowledge is important to tailor interventions and information. 


\section{Introduction}

Increased long-term survival after lung cancer has led to a growing interest in addressing health-related quality of life (HRQoL) and symptoms faced by long-term survivors (1). Surgery is the most important treatment modality to improve survival in patients with lung cancer and is the treatment of choice for patients with early stage, non-small-cell lung cancer (NSCLC) (2). However, surgery may affect the patients' HRQoL (3) and the development of chronic pain after surgery, also called persistent postsurgical pain (PPSP), is a common complication following thoracic surgery (4-6). PPSP is defined as pain that develops after a surgical procedure, persists more than $2-3$ months postsurgery without other causes, and has different characteristics from preoperative pain $(6,7)$. PPSP can interfere with daily activities, sleep, mood, and HRQoL $(8,9)$, hence being a clinical relevant problem delaying recovery after surgery (10). Poorly managed postoperative pain may delay discharge from hospital and results in the patient's inability to participate in rehabilitation programs, leading to poor outcomes also after the initial postoperative period (11).

Thoracic surgery is among the surgical procedures associated with the highest prevalence of PPSP reported in 19-60\% of the patients after lung cancer surgery (12-16). Previous studies have identified younger age $(12,16,17)$, female gender $(17)$, preoperative pain (18), and anxiety and depression (8) as predictors for PPSP after thoracic surgery. Despite recent advances in surgical techniques and perioperative care, such as the introduction of video-assisted thoracic surgery (VATS) and enhanced recovery pathways, PPSP remains an important clinical issue in thoracic surgery and has not decreased notably $(14,19-21)$.

Patients with lung cancer not only experience the surgical trauma of their disease treatment but also experience several physiological, psychological and social symptoms and 
changes because of the disease process $(22,23)$. However, reports on pain following lung cancer surgery remain scarce or exhibit several methodological shortcomings $(12,20,24)$, in particular regarding the heterogeneity of the studied populations $(12,13)$, the retrospective character of analyses $(17,18)$, the absence of standardized evaluation procedures $(25)$, and differences in the timing of follow-up (14). Previous prospective studies have mainly followed pain trajectories over a short time and/or often lack baseline measures. Further, we have not found any published studies that have attempted to identify subgroups of patients based on differences in pain trajectories following lung cancer surgery.

Given the limitations noted above, here we aimed to describe the pain trajectory in a cohort of patients undergoing surgery for primary lung cancer. Further, we wished to investigate whether distinct groups of patients could be identified based on different pain trajectories, and — if such groups could be identified — to compare the demographical and clinical characteristics between them.

\section{Methods}

\section{Patients, settings, and study procedures}

This exploratory study is based on secondary analyses of data from a larger longitudinal study of symptoms in patients with lung cancer before and after surgery $(26,27)$. Patients scheduled for surgery who were 18 years or older, and were able to read, write, and understand Norwegian, were eligible for inclusion. Exclusion criteria were cognitive impairment, cancellation of surgery, surgical exploration without curative resection, or cases where histopathology after surgery revealed that the patients had benign or metastatic disease.

The patients were recruited at three university hospitals in Norway (Oslo University Hospital, St. Olav's Hospital and Haukeland University Hospital). The study was approved by 
the Regional Ethical Review Committee (REK number 2010/1508) and supported by the Institutional Review Boards at all hospitals involved.

Most patients (91\%) were recruited in the hospital 1-3 days before surgery. The remaining $9 \%$ of the patients were recruited in the outpatient clinic prior to surgery. After giving written informed consent, patients completed baseline study questionnaires before surgery. Data were also collected by postal surveys at 1, 5, 9 and 12 months after surgery. Nonresponders were sent one postal reminder after the initial questionnaire. The recruitment started in November 2010 and was completed in March 2012.

\section{Data collection}

\section{Demographic and clinical characteristics}

Patients provided information on gender, living situation, level of education, and employment status. Research nurses completed information on age, smoking status, lung function, height, weight, and preoperative medications for all patients. After surgery, the patients' medical records were reviewed for information on tumor histology, type of surgery, and TNM classification (28). The TNM classification is a system for cancer staging based on tumor (T), node $(\mathrm{N})$ and metastasis $(\mathrm{M})$.

\section{The Brief Pain Inventory (BPI)}

The primary outcome was pain intensity, measured by BPI average pain intensity and BPI worst pain intensity (29). BPI is a short, self-administered questionnaire designed to assess pain occurrence, intensity, interference with function, pain relief, and pain localization. Only pain intensity (average and worst pain) and pain localization were used in this study. Patients were asked to indicate whether they had pain (yes/no). If they had pain, they rated the severity of their average and worst pain using a 0 (no pain) to 10 (pain as bad as you can imagine) numerical rating scale (NRS). Different cut-points have been recommended for categorizing pain intensity into subcategories (30). We defined mild pain as NRS $1-3$, moderate pain as 4- 
6, and severe pain as 7-10 (31). The Norwegian version of BPI has shown well-established validity, reliability, and sensitivity to change $(32,33)$.

\section{The Self-administered Comorbidity Questionnaire-19 (SCQ-19)}

Comorbidities were assessed by the SCQ-19, which includes 16 common comorbidities and three optional conditions. Patients indicated whether they had the comorbid condition (yes/no); if they received treatment for it (yes/no); and whether it limited their activities (yes/no). The total SCQ-19 score can range from 0 to 57: a higher total score indicates a more severe comorbidity profile. The SCQ has well-established validity and reliability in patients with chronic conditions $(34,35)$. In this study, only the total number of comorbidities (0-19) and the occurrence of the most common pain conditions were used in the analyses.

\section{Statistical analyses}

Statistical analyses were performed using IBM SPSS Statistics (v.23.0; IBM Corp, Armonk, NY, USA) and the R statistical program (36).

Data have been were summarized as means ( \pm standard deviations), medians (percentiles) or frequencies (percentages), as appropriate. The pain profiles for average and worst pain were analyzed using latent class mixed models (LCMMs), implemented in the $\mathrm{R}$ package $l \mathrm{cmm}$ v. 1.7.7 (37). Such models can be used to identify unmeasured, latent classes that represent subgroups of patients with similar symptom profiles across time. These subgroups can then be compared with respect to sociodemographic and clinical variables. The analyses were performed separately for "average pain" and "worst pain". Patients who did not report any pain at any of the five occasions (i.e., pain score $=0$ at all time points) were considered to belong to a predefined "no pain class" and were not included in the LCMM analysis.

The pain profiles were specified in terms of a linear mixed model (LMM) for an unobservable, latent variable, assumed to be normally distributed. The LMM included 
variables representing the time profile as the only fixed effects variables, and a random intercept to account for within-subject correlations. The pain scores, observed on an ordinal scale of $0-10$, were linked to the unobserved, latent variable by a probit link, assuming that the cumulative probabilities for the pain scores depended on the latent pain profiles.

A structure for the latent, class-specific time profiles was first selected for a one-class model. The candidate models were polynomials up to the second order, and two models with a categorical time variable: a two-category model with a temporary shift at 1 month after surgery but an otherwise constant time profile, and a three-category model for which the profile after 1 month was allowed to return to a level different from the baseline level. After deciding on the time structure for the one-class model, based on tests for significance for the model parameters, models with one to four classes were fitted using the selected time structure. The final number of classes was decided to be the one with the smallest value of the Bayesian Information Criterion (BIC) (38). The parameter estimation was performed using an iterative procedure in a maximum-likelihood framework. The gridsearch function in the $l \mathrm{cmm}$ package was used to obtain initial values for the parameters.

Each patient was allocated to the class for which the patient's estimated membership probability was the largest. Sociodemographic and clinical variables, chosen based on clinical relevance and previous research, were compared between the classes by analysis of variance (ANOVA), Kruskal-Wallis tests, Chi-squared tests, or Fisher's exact tests, as appropriate. $P$ values $<0.05$ were considered statistically significant. The Holm-Bonferroni correction method was applied to adjust for multiple comparisons.

No sample size calculations were performed for the secondary analyses reported in this exploratory study. 


\section{Results}

A total of 375 patients with presumptive primary lung cancer were asked to participate in the study, and 307 agreed (81\%). Twenty-two patients were excluded from the study because of metastatic tumors, benign disease, or cancelled or exploratory surgery. Of the remaining 285 patients, 264 completed the questionnaire at baseline (93\%). Figure 1 shows the inclusion and exclusion of patients in the entire study.

\section{Patient characteristics}

Demographic and clinical characteristics of the total sample at baseline are listed in Table 1. The sample consisted of $152(58 \%)$ men and 112 (42\%) women; the mean age was $65.8( \pm$ 8.5) years, and the age range was $30-87$ years. The mean number of comorbidities measured by the SCQ-19 was $2.4( \pm 1.7)$, range $0-8$. The most common comorbid conditions were back/neck pain (42\%), high blood pressure (31\%), other lung disease (29\%), and osteoarthritis $(24 \%)$.

The majority of the patients were staged with cancer TNM I/II (81\%), while most patients had an adenocarcinoma (56\%) or a squamous-cell carcinoma (31\%). The surgical approach ranged from lobectomy (69\%) to bilobectomy (7\%), pulmectomy (9\%), wedge resection (9\%), and VATS (5\%). Psychotropic drugs were used by $20 \%$ of the patients, while $16 \%$ used pain medication before surgery.

\section{Pain occurrence for the total sample}

In total, the percentage of patients reporting occurrence of any pain increased from $40 \%$ before surgery to a peak of $69 \%$ after 1 month, and decreased to $56 \%$ at 5 months, $57 \%$ at 9 months, and $55 \%$ at 12 months. Pain was reported both at baseline and 12 months by $29 \%$ of the patients. The proportions of patients reporting none (0), mild (1-3), moderate (4-6) and severe pain (7-10) at each assessment are illustrated in Figure 2A for average pain and Figure 
2B for worst pain. The observed proportion of patients with moderate pain was highest after 1 month, reduced after 5 and 9 months and increased after 12 months, for both average and worst pain. Pain was reported at different sites during the 1-year trajectory (Supplementary Table S1). Back (22\%), neck/shoulder (20\%), and hips (13\%) were the most common sites before surgery, while chest (43\%), neck/shoulders (36\%), and back (32\%) were the most common after 1 month. Upper back pain was reduced to baseline level after 5, 9, and 12 months, while chest pain and pain in neck/shoulders were most frequently reported up to 12 months after surgery.

\section{Pain trajectory for average and worst pain intensity}

The pain trajectories of both average and worst pain showed substantial inter-individual variations over the five time points as illustrated by average pain in Figure 3.

\section{Average pain intensity}

The results from the LCMM analysis using one to four classes are summarized in Table 2A. A model with two classes gave the best fit in terms of the smallest BIC value among the candidate models. The estimated pain trajectories for the two-class model, with the constant trajectory for the "no pain" class inserted, are illustrated in Figure 4A. The "no pain" trajectory comprised 21 patients (8\%), not included when fitting the LCMM. One of the two trajectories (Class 1) estimated by the LCMM indicated a postoperative elevation of pain at 1 month, with a reduction of the pain at 5 months and a stable trajectory at 5-12 months. The other estimated trajectory (Class 2) indicated a stable pain profile, but a higher baseline pain score than for Class 1.

\section{Worst pain intensity}

Similar results are shown for "worst pain" in Table 2B, where a model with two classes gave the best fit in terms of the smallest BIC value among the candidate models. The estimated 
pain trajectories for the two-class model, with the constant trajectory for the "no pain" class ( $n$ $=21$ ), inserted, are illustrated in Figure 4B. The plot shows that Class 1 started low with high ratings after 1 month, then returned to a level slightly higher than the baseline scores. Class 2 started higher but typically had similar scores through the whole trajectory.

\section{Differences between the pain classes in patients' demographic and clinical}

\section{characteristics}

Sociodemographic and clinical variables were compared between the identified classes fitted in the LCMMs. The "no pain" class was included as a third class in the comparisons. Tables 3A and 3B show univariate analyses comparing the classes for "average pain" and "worst pain", respectively. After adjusting for multiple testing, comorbidity, preoperative use of pain medicine, and type of surgery differed significantly between the classes both for "average" and "worst pain". Higher comorbidity scores and preoperative use of pain medicine characterized the class with overall highest pain both for "average" and "worst pain". Concerning the type of surgery, lobectomy was more frequent among the patients in the two pain classes, while the percentages of wedge resection and VATS were highest in the "no pain" class for both "average" and "worst pain". For "average pain", there was also a significant difference in the preoperative use of psychotropic medicine, which was more common in the class of patients with the highest pain. Patients in the "no pain" class were observed to be older than patients in the two other pain classes, and smoking until surgery was more prevalent by increasing average pain, but these results were not statistically significant.

\section{Discussion}

Our study is the first to use LCMMs to identify subgroups of patients with distinct pain trajectories following surgery for lung cancer. Two distinct classes for "average" and "worst pain" were identified over time and the patterns were similar for both items. The estimated 
pain trajectory for the first class started low before surgery with increased ratings after 1 month, then returned to a stable level slightly higher than the baseline scores. The pain trajectory for the second class started at a higher level of pain but stayed relatively stable throughout the whole trajectory. In addition, $8 \%$ of the patients did not report having pain during the 1-year period.

\section{Pain intensity before the surgery}

Pain was reported by $40 \%$ of the patients before surgery. This level is higher than in the Norwegian general population that reports $24.4-30 \%(39,40)$. One reason for this could be that our sample had a mean age of 66 years, and pain is more common in the elderly (41). As pain is usually not a symptom of early stage lung cancer (42), the high proportion of pain reported at baseline might represent comorbid conditions. Of note, $42 \%$ of the sample reported back/neck pain, and $24 \%$ reported osteoarthritis before surgery. This is line with Hetmann et al. (43) who reported that most commonly patients reported pain from the neck, shoulders, lower back, hips, or knees before surgery. Wildgaard et al. also found that patients with post-thoracotomy pain syndrome commonly experienced pain in other body parts (9), while Fagundes et al. (44) found that patients who reported any comorbid condition before surgery reported higher levels of postoperative pain. This supports the idea that in general, preoperative pain is a risk factor for developing persistent pain after surgery (45). Pain before surgery might also be increased by the stress of undergoing major surgery and being diagnosed with a life-threatening and serious disease like lung cancer.

\section{Variation in pain over time}

This study showed that there was a high occurrence of pain preoperatively as well as at 1,5 , 9, and 12 months after surgery for lung cancer. High prevalence rates of persistent pain were reported in several previous studies after thoracic surgery $(8,14,15,20)$. The proportion of 
patients reporting the occurrence of pain in this study increased after 12 months compared with baseline for the whole sample and the pain trajectories showed that the pain intensity was higher after surgery for some patients. Overall, this supports previous reports on the high prevalence of persistent pain after surgery for lung cancer (21). We assume the pain is both postoperative pain and pain from comorbidities as the levels of — for exampleneck/shoulder and back pain were high during the whole trajectory. However, inter-individual variations and the identification of different pain classes confirmed that these patients followed different pain trajectories.

The LCMM analysis revealed that patients with pain at 5 months still experienced pain at 12 months. The high frequency of pain located in the chest, neck, and shoulder area after 12 months suggests that pain was not only explained by high comorbidity. Persistent pain after lung cancer surgery might add to the burden of symptoms directly linked to the cancer diagnosis. Further, it might impact on postoperative recovery and influence complications. Recovery after oncology surgery is further complicated by potential changes caused by adjuvant therapy (46). Pain after lung cancer surgery is indeed a complicated interplay between changes related to the operation, changes related to the disease, and comorbid conditions.

\section{Vulnerable patients}

Our study identified different classes of pain trajectories and described these subgroups in terms of demographic and clinical variables. Consistent with previous studies $(12,16,17)$ patients in the two pain classes were younger than patients with no pain. They had more comorbidities, more prevalent use of pain and psychotropic medicines, and smoked more frequently until surgery. These findings support the idea that patients with high pain during their trajectory can be described as a vulnerable group. This information could be used to 
target information to patients, as well as to enable more individualized pain regimen as part of patient-centered care.

The proportions of women were highest in the classes with pain, but gender was not significantly related to the pain class. This finding was unexpected, because several studies have revealed that women have a higher risk of both acute and long-term pain after lung cancer surgery $(47,48)$. The proportion of women included in our study was high $(42 \%)$ but it is possible that the power to identify gender differences was too weak. Moreover, it cannot be ruled out that men and women differ regarding factors important for pain not identified in our study. Further research on possible gender differences in postsurgical pain is needed.

\section{Strengths and limitations}

The key strengths of this study were the prospective design with baseline measures of pain, the large number of participants, the high response rate, the consecutive inclusion, and the homogeneous sample including only patients with primary lung cancer. Further, this was a multicenter study with patient inclusion from four out of seven centers performing lung cancer surgery in Norway. We consider this cohort to be representative of patients with lung cancer receiving surgical treatment in Norway at the time of the study. However, some limitations need to be discussed.

First, patients were not asked about the etiology of their pain. Hence, we do not know whether the pain could be classified as postsurgical pain, was related to the treatment for lung cancer (adjuvant treatment/chemo), was related to comorbidities, progression or recurrence of lung cancer. However, the increase in pain after 1 month in one class of patients suggests that pain related to surgery was captured. Second, most of the patients were subjected to thoracotomy, while VATS has become the treatment of choice in many cardiothoracic centers (48). VATS is less invasive and has been thought to change the pattern of symptoms and pain. 
However, even with the VATS approach, pain is still an important clinical issue in lung cancer surgery $(20,49)$.

Patients reporting no pain at all five occasions were excluded from the LCMM analysis and were predefined to belong to a separate "no pain" class. This approach has been taken by other studies identifying latent classes of pain $(50,51)$. Rerunning the analysis using all data gave similar profiles for the two-class model to the ones illustrated in Figure 4. However, when including patients not reporting any pain, a one-class model gave better fit based on the BIC, most likely because of increased uncertainty for the estimated profiles. One of the strengths of the LCMM approach is that all observations for each patient were used, avoiding listwise deletion.

\section{Conclusion}

Our study showed that pain was highly prevalent during the first year after surgery for lung cancer, and subgroups of patients could be identified based on different pain trajectories. Importantly, high levels of pain at baseline illustrated that comorbidities and preoperative use of pain medicine were associated with having pain at 1 year after surgery. That so many patients awaiting lung cancer surgery had chronic pain-related comorbidities must be taken into consideration when estimating the postsurgery pain levels and when deciding pain treatment for these patients. Further, one must be aware that these patients experience both postoperative pain and pain from chronic conditions. Knowledge about vulnerable patient groups and risk factors for pain is important to tailor interventions and information about pain after lung cancer surgery. 


\section{Disclosures and Acknowledgements}

The study was funded by the Norwegian Cancer Society.

The authors have no conflicts of interest to declare. 


\section{References}

1. Gooneratne NS, Dean GE, Rogers AE, et al. Sleep and quality of life in long-term lung cancer survivors. Lung Cancer 2007;58:403-10.

2. Manser R, Wright G, Hart D, Byrnes G, Campbell DA. Surgery for early stage nonsmall cell lung cancer. Cochrane Database Syst Rev2005(1):Cd004699.

3. Kenny PM, King MT, Viney RC, et al. Quality of life and survival in the 2 years after surgery for non small-cell lung cancer. J Clin Oncol 2008;26:233-41.

4. Kehlet H, Jensen TS, Woolf CJ. Persistent postsurgical pain: risk factors and prevention. Lancet 2006;367:1618-25.

5. Wildgaard K, Kehlet H. Chronic post-thoracotomy pain - What is new in pathogenic mechanisms and strategies for prevention? Tech Reg Anesth Pain Manag 2011;15:83-9.

6. Richebe P, Capdevila X, Rivat C. Persistent postsurgical pain: pathophysiology and preventative pharmacologic considerations. Anesthesiol 2018;129:590-607.

7. Macrae WA. Chronic post-surgical pain: 10 years on. Br J Anaesth 2008;101:77-86.

8. Hopkins KG, Hoffman LA, Dabbs Ade V, et al. Postthoracotomy pain syndrome following surgery for lung cancer: symptoms and impact on quality of life. J Adv Pract Oncol $2015 ; 6: 121-32$.

9. Wildgaard K, Ravn J, Nikolajsen L, et al. Consequences of persistent pain after lung cancer surgery: a nationwide questionnaire study. Acta Anaesthesiol Scand 2010;55:60-8.

10. Althaus A, Hinrichs-Rocker A, Chapman R, et al. Development of a risk index for the prediction of chronic post-surgical pain. Eur J Pain 2012;16:901-10.

11. Rawal N. Current issues in postoperative pain management. Eur J Anaesthesiol 2016;33:160-71. 
12. Wildgaard K, Ravn J, Kehlet H. Chronic post-thoracotomy pain: a critical review of pathogenic mechanisms and strategies for prevention. Eur J Cardiothorac Surg 2009;36:17080.

13. Grosen K, Laue Petersen G, Pfeiffer-Jensen M, Hoejsgaard A, Pilegaard HK.

Persistent post-surgical pain following anterior thoracotomy for lung cancer: a cross-sectional study of prevalence, characteristics and interference with functioning. Eur J Cardiothorac Surg 2013;43:95-103.

14. Bayman EO, Brennan TJ. Incidence and severity of chronic pain at 3 and 6 months after thoracotomy: meta-analysis. J Pain 2014;15:887-97.

15. Bayman EO, Parekh KR, Keech J, Selte A, Brennan TJ. A prospective study of chronic pain after thoracic surgery. Anesthesiol 2017;126:938-51.

16. Mongardon N, Pinton-Gonnet C, Szekely B, et al. Assessment of chronic pain after thoracotomy: a 1-year prevalence study. Clin J Pain 2011;27:677-81.

17. Peng Z, Li H, Zhang C, et al. A retrospective study of chronic post-surgical pain following thoracic surgery: prevalence, risk factors, incidence of neuropathic component, and impact on quality of life. PLoS One 2014;9:e90014.

18. Shanthanna H, Aboutouk D, Poon E, et al. A retrospective study of open thoracotomies versus thoracoscopic surgeries for persistent postthoracotomy pain. J Clin Anesth 2016;35:215-20.

19. Bendixen M, Jorgensen OD, Kronborg C, Andersen C, Licht PB. Postoperative pain and quality of life after lobectomy via video-assisted thoracoscopic surgery or anterolateral thoracotomy for early stage lung cancer: a randomised controlled trial. Lancet Oncol 2016;17:836-44. 
20. Rizk NP, Ghanie A, Hsu M, et al. A prospective trial comparing pain and quality of life measures after anatomic lung resection using thoracoscopy or thoracotomy. Ann Thorac Surg 2014;98:1160-6.

21. Rodriguez-Aldrete D, Candiotti KA, Janakiraman R, Rodriguez-Blanco YF. Trends and new evidence in the management of acute and chronic post-thoracotomy pain-an overview of the literature from 2005 to 2015. J Cardiothorac Vasc Anesth 2016;30:762-72. 22. Hopkins KG, Rosenzweig M. Post-thoracotomy pain syndrome: assessment and intervention. Clin J Oncol Nurs 2012;16:365-70.

23. Lowery AE, Krebs P, Coups EJ, et al. Impact of symptom burden in post-surgical nonsmall cell lung cancer survivors. Support Care Cancer 2014;22:173-80.

24. Montes A, Sabate S, Roca G, Canet J. Preoperative prediction of chronic postsurgical pain after thoracotomy: need for adequately sized population-based samples. Anesthesiol 2018;128:224.

25. Guastella V, Mick G, Soriano C, et al. A prospective study of neuropathic pain induced by thoracotomy: Incidence, clinical description, and diagnosis. Pain 2011;152:74-81. 26. Oksholm T, Miaskowski C, Kongerud JS, et al. Does age influence the symptom experience of lung cancer patients prior to surgery? Lung Cancer 2013;82:156-61.

27. Oksholm T, Rustoen T, Cooper B, et al. Trajectories of symptom occurrence and severity from before through five months after lung cancer surgery. J Pain Symptom Manage 2015;49:995-1015.

28. Goldstraw P (Ed.). International Association for the Study of Lung Cancer (IASLC) staging manual in thoracic oncology. Orange Park, FL: Editorial Rx Press, 2009.

29. Cleeland CS, Ryan KM. Pain assessment: global use of the Brief Pain Inventory. Ann Acad Med Singapore 1994;23:129-38. 
30. Kapstad H, Hanestad BR, Langeland N, Rustoen T, Stavem K. Cutpoints for mild, moderate and severe pain in patients with osteoarthritis of the hip or knee ready for joint replacement surgery. BMC Musculoskelet Disord 2008;9:55.

31. Lahtinen P, Kokki H, Hynynen M. Pain after cardiac surgery: a prospective cohort study of 1-year incidence and intensity. Anesthesiol 2006;105:794-800.

32. Klepstad P, Loge JH, Borchgrevink PC, et al. The Norwegian brief pain inventory questionnaire: translation and validation in cancer pain patients. J Pain Symptom Manage 2002;24:517-25.

33. Gjeilo KH, Stenseth R, Wahba A, Lydersen S, Klepstad P. Validation of the brief pain inventory in patients six months after cardiac surgery. J Pain Symptom Manage 2007;34:64856.

34. Charlson ME, Pompei P, Ales KL, MacKenzie CR. A new method of classifying prognostic comorbidity in longitudinal studies: development and validation. J Chronic Dis 1987;40:373-83.

35. Sangha O, Stucki G, Liang MH, Fossel AH, Katz JN. The Self-Administered Comorbidity Questionnaire: a new method to assess comorbidity for clinical and health services research. Arthrit Rheumat 2003;49:156-63.

36. R Core Team. R: A language and environment for statistical computing. Vienna, Austria: R Foundation for Statistical Computing, 2018.

37. Proust-Lima C, Philipps V, Liquet B. Estimation of Extended Mixed Models Using Latent Classes and Latent Processes: The R Package lcmm. J Stat Softw 2017;78:1-56.

38. Schwarz G. Estimating the dimension of a model. Ann Stats 1978;6:461-4.

39. Rustoen T, Wahl AK, Hanestad BR, et al. Prevalence and characteristics of chronic pain in the general Norwegian population. Eur J Pain 2004;8:555-65. 
40. Landmark T, Romundstad P, Dale O, Borchgrevink PC, Kaasa S. Estimating the prevalence of chronic pain: validation of recall against longitudinal reporting (the HUNT pain study). Pain 2012;153:1368-73.

41. Landmark T, Romundstad P, Dale O, et al. Chronic pain: One year prevalence and associated characteristics (the HUNT pain study). Scand J Pain 2013;4:182-7.

42. Postmus PE, Kerr KM, Oudkerk M, et al. Early and locally advanced non-small-cell lung cancer (NSCLC): ESMO Clinical Practice Guidelines for diagnosis, treatment and follow-up. Ann Oncol 2017;28(Suppl. 4):iv1-iv21.

43. Hetmann F, Schou-Bredal I, Sandvik L, Kongsgaard UE. Does chronic pre-operative pain predict severe post-operative pain after thoracotomy? A prospective longitudinal study. Acta Anaesthesiol Scand 2013;57:1065-72.

44. Fagundes CP, Shi Q, Vaporciyan AA, et al. Symptom recovery after thoracic surgery: Measuring patient-reported outcomes with the MD Anderson Symptom Inventory. J Thorac Cardiovasc Surg 2015;150:613-9.e2.

45. Gjeilo KH, Stenseth R, Klepstad P. Risk factors and early pharmacological interventions to prevent chronic postsurgical pain following cardiac surgery. Am J Cardiovas Drugs 2014;14:335-42.

46. Lee L, Tran T, Mayo NE, Carli F, Feldman LS. What does it really mean to "recover" from an operation? Surgery 2014;155:211-6.

47. Ochroch EA, Gottschalk A, Troxel AB, Farrar JT. Women suffer more short and longterm pain than men after major thoracotomy. Clin J Pain 2006;22:491-8.

48. Kwon ST, Zhao L, Reddy RM, et al. Evaluation of acute and chronic pain outcomes after robotic, video-assisted thoracoscopic surgery, or open anatomic pulmonary resection. $\mathrm{J}$ Thorac Cardiovasc Surg 2017;154:652-9.e1. 
49. Wildgaard K, Ringsted TK, Hansen HJ, Petersen RH, Kehlet H. Persistent postsurgical pain after video-assisted thoracic surgery-an observational study. Acta Anaesthesiol Scand 2016;60:650-8.

50. Bentsen SB, Miaskowski C, Cooper BA, Christensen VL, Henriksen AH, Holm AM, et al. Distinct pain profiles in patients with chronic obstructive pulmonary disease. Int $\mathbf{J}$ Chronic Obst Pulmon Dis 2018;13:801-11.

51. Miaskowski C, Paul SM, Cooper B, et al. Identification of patient subgroups and risk factors for persistent arm/shoulder pain following breast cancer surgery. Eur J Oncol Nurs $2014 ; 18: 242-53$. 


\section{Figure legends}

Figure 1. Flow chart of the patients enrolled in the study

Figure 2A. The observed proportion (\%) of patients reporting no pain, mild pain, moderate pain, or severe pain at each time point for the average pain item.

Figure 2B. The observed proportion (\%) of patients reporting no pain, mild pain, moderate pain, or severe pain at each time point for the worst pain item.

Figure 3. The patients' individual pain trajectories over the five time points for average pain. Figure 4A. The estimated trajectories from the two-class latent class mixed model (LCMM) for average pain, with the constant trajectory for the "no pain" class inserted.

The plot shows the median and the 2.5- and 97.5 - percentiles of the posterior distribution for the predicted trajectories from the LCMM analysis. The numbers of patients $(n)$ in Classes 1 and 2 refer to the numbers predicted by the LCMM to belong to each class.

Figure 4B. The estimated trajectories from the two-class latent class mixed model (LCMM) for worst pain with the constant trajectory for the "no pain" class inserted.

The plot shows the median and the 2.5- and 97.5-percentiles of the posterior distribution for the predicted trajectories from the LCMM analysis. The numbers of patients $(n)$ in Classes 1 and 2 refer to the numbers predicted by the LCMM to belong to each class. 
Table 1. Demographic and clinical characteristics of the patients with lung cancer before surgery.

Characteristic $\left(n=245-264^{\mathrm{a}}\right)$

$n(\%)$ or mean $( \pm \mathrm{SD})$

Age (years)

$65.8( \pm 8.5)$

Female gender

$112(42.4)$

Living alone

$59(23.5)$

Educational level:

Primary / secondary school

$211(83.7)$

University / college

$41(16.3)$

Employment status:

Full- or part-time

75 (29.9)

Sick leave or disability

$66(26.3)$

Retired or other

$110(43.8)$

Smoking status:

Smoked until surgery

$120(45.6)$

Stopped until at least 1 year before surgery

$126(47.9)$

Never smoked

$17(6.5)$

BMI in $\mathrm{kg} / \mathrm{m}^{2}$

$25.9( \pm 4.7)$

Tumor type:

Adenocarcinoma

$149(56.4)$

Squamous cell carcinoma

$81(30.7)$

Small cell

$6(2.3)$

Carcinoid

$6(2.3)$

Other

$22(8.3)$ 
TNM stage of cancer

IA

$80(32.7)$

IB

79 (29.9)

II

$45(18.4)$

IIIA

$40(16.4)$

IIIB

$1(0.4)$

Psychotropic drugs before surgery (yes)

$53(20.1)$

Pain medication before surgery (yes)

$42(15.9)$

Number of comorbidities measured by SCQ- $19^{\mathrm{b}}$

$2.4(1.7)$

Back/neck pain

$111(42.0)$

High blood pressure

$82(31.1)$

Other lung disease (including COPD)

$76(28.8)$

Osteoarthritis, degenerative arthritis

$62(23.5)$

Heart disease

$46(17.4)$

Depression

$29(11.0)$

Headaches

$28(10.6)$

Dermatological diseases

$25(9.5)$

Rheumatoid arthritis

$24(9.1)$

Diabetes

$18(6.8)$

Disease of connective tissues or muscle

$13(4.9)$

Ulcers or stomach disease

$11(4.2)$

Intestinal disease

$10(3.8)$

Kidney disease

$7(2.7)$

Anemia or other blood disorder

$1(0.4)$

Liver disease

$1(0.4)$ 
COPD, chronic obstructive pulmonary disease; SCQ, Self-Administered Comorbidity Questionnaire; TNM, tumor-node-metastasis; BMI, body mass index; SD, standard deviation of the mean.

${ }^{\mathrm{a}} n$ values vary because of missing values. ${ }^{\mathrm{b}} \mathrm{SCQ}$ includes 16 common comorbidities and three optional conditions. 
Table 2A. Results from the latent class mixed model and fit indices for the outcome variable "average pain."

\begin{tabular}{lllll}
\hline & Log- & No. of & BIC & AIC \\
Model & likelihood & parameters & & \\
\hline 1 Class & -1750.62 & 13 & 3572.65 & 3527.24 \\
2 Class & $\mathbf{- 1 7 3 5 . 3 2}$ & $\mathbf{1 7}$ & $\mathbf{3 5 6 4 . 0 3}$ & $\mathbf{3 5 0 4 . 6 5}$ \\
3 Class & -1726.57 & 21 & 3568.49 & 3495.13 \\
4 Class & -1722.23 & 25 & 3581.79 & 3494.47 \\
\hline
\end{tabular}

AIC, Akaike's Information Criterion; BIC, Bayesian Information Criterion.

The model with the best fit in bold (i.e. the lowest BIC).

Table 2B. Results from the latent class mixed model and fit indices for the outcome variable "worst pain."

\begin{tabular}{lllll}
\hline & Log- & No. of & BIC & AIC \\
Model & likelihood & parameters & & \\
\hline 1 Class & -1791.13 & 13 & 3653.67 & 3608.26 \\
2 Class & $\mathbf{- 1 7 7 7 . 1 1}$ & $\mathbf{1 7}$ & $\mathbf{3 6 4 7 . 6 1}$ & $\mathbf{3 5 8 8 . 2 3}$ \\
3 Class & -1771.62 & 21 & 3658.60 & 3585.25 \\
4 Class & -1767.49 & 25 & 3672.30 & 3584.97 \\
\hline
\end{tabular}

AIC, Akaike's Information Criterion; BIC, Bayesian Information Criterion

The model with the best fit in bold (i.e. the lowest BIC). 
Table 3A. Univariate analyses comparing sociodemographic and clinical variables in pain classes for average pain.

\begin{tabular}{|c|c|c|c|c|c|}
\hline & $\begin{array}{l}\text { No pain } \\
(n=21)\end{array}$ & $\begin{array}{l}\text { Class } 1 \\
(n=169)\end{array}$ & $\begin{array}{l}\text { Class } 2 \\
(n=74)\end{array}$ & $P$ & $\begin{array}{l}\text { Adjusted } \\
P^{e}\end{array}$ \\
\hline Age, years, mean $( \pm S D)$ & $70.7(6.5)$ & $65.8(8.1)$ & $64.3(9.4)$ & $0.009^{a}$ & 0.159 \\
\hline $\mathrm{BMI} \mathrm{kg} / \mathrm{m}^{2}$, mean $( \pm \mathrm{SD})$ & $24.8(4.3)$ & $25.9(4.8)$ & $26.0(4.7)$ & $0.583^{\mathrm{a}}$ & 1.000 \\
\hline $\begin{array}{l}\text { SCQ-19 total, median (Q1- } \\
\text { Q3) }\end{array}$ & $2.0(1.0-2.2)$ & $2.0(1.0-3.0)$ & $3.0(2.0-4.2)$ & $<0.001^{b}$ & $<0.001$ \\
\hline $\begin{array}{l}\text { Pack-years smoking, median } \\
\text { (Q1-Q3) }\end{array}$ & $20.0(13.9-34.4)$ & $30.0(18.4-40.6)$ & $28.0(18.6-44.5)$ & $0.486^{\mathrm{b}}$ & 1.000 \\
\hline $\begin{array}{l}\text { Days with chest-tube, median } \\
\text { (Q1-Q3) }\end{array}$ & $2(1-4)$ & $3(2-5)$ & $3(2-5)$ & $0.370^{\mathrm{b}}$ & 1.000 \\
\hline Gender, $n(\%)$ & & & & $0.472^{\mathrm{c}}$ & 1.000 \\
\hline Female & $7(33.3)$ & $70(41.4)$ & $35(47.3)$ & & \\
\hline Cancer type, $n(\%)$ & & & & $0.235^{\mathrm{c}}$ & 1.000 \\
\hline Adenocarcinoma & $9(42.9)$ & $97(57.4)$ & $43(58.1)$ & & \\
\hline Squamous cell & $11(52.4)$ & $47(27.8)$ & $23(31.1)$ & & \\
\hline
\end{tabular}




\begin{tabular}{|c|c|c|c|c|c|}
\hline Other & $1(4.8)$ & $25(14.8)$ & $8(10.8)$ & & \\
\hline Psychotropic medicine, $n(\%)$ & & & & $<0.001^{c}$ & $<0.001$ \\
\hline Yes & $2(9.5)$ & $21(12.4)$ & $30(40.5)$ & & \\
\hline Any pain medicine, $n(\%)$ & & & & $<0.001^{\mathrm{c}}$ & $<0.001$ \\
\hline Yes & $1(4.8)$ & $15(8.9)$ & $26(35.1)$ & & \\
\hline TNM stage of cancer, $n(\%)$ & & & & $0.775^{\mathrm{c}}$ & 1.000 \\
\hline IA & $4(21.1)$ & $55(35.0)$ & $21(30.4)$ & & \\
\hline IB & $8(42.1)$ & $49(31.2)$ & $22(31.9)$ & & \\
\hline II & $5(26.3)$ & $26(16.8)$ & $14(20.3)$ & & \\
\hline IIIA/IIIB & $2(10.5)$ & $27(17.2)$ & $12(17.4)$ & & \\
\hline Smoking group, $n(\%)$ & & & & $0.021^{d}$ & 0.362 \\
\hline Smoked until surgery & $6(28.6)$ & $70(41.4)$ & $44(60.3)$ & & \\
\hline $\begin{array}{l}\text { Stopped } 1 \text { year before } \\
\text { surgery }\end{array}$ & $14(66.7)$ & $85(50.3)$ & $27(37.0)$ & & \\
\hline Never smoked & $1(4.8)$ & $14(8.3)$ & $2(2.7)$ & & \\
\hline Surgery type, $n(\%)$ & & & & $<0.001^{d}$ & $<0.001$ \\
\hline Lobectomy & $5(23.8)$ & $133(79.2)$ & $45(60.8)$ & & \\
\hline
\end{tabular}




\begin{tabular}{|c|c|c|c|c|c|}
\hline Bilobectomy & $2(9.5)$ & $12(7.1)$ & $5(6.8)$ & & \\
\hline Pulmectomy & $4(19.0)$ & $10(6.0)$ & $9(12.2)$ & & \\
\hline Wedge resection & $7(33.3)$ & $8(4.8)$ & $9(12.2)$ & & \\
\hline Thoracoscopic & $3(14.3)$ & $5(3.0)$ & $6(8.1)$ & & \\
\hline Additional surgery, $n(\%)$ & & & & $0.450^{\mathrm{c}}$ & 1.000 \\
\hline Yes & $1(4.8)$ & $24(14.2)$ & $12(16.2)$ & & \\
\hline
\end{tabular}

BMI, body mass index; SCQ-19, Self-Administered Comorbidity Questionnaire; Q1, lower quartile $\left(25^{\text {th }}\right.$ percentile); Q3, upper quartile (75 ${ }^{\text {th }}$ percentile). Classes identified by latent class mixed model (LCMM); No pain class; Class $1=$ elevated pain; Class $2=$ stable pain. ${ }^{a}$ ANOVA, ${ }^{\mathrm{b}}$ Kruskal-Wallis, ${ }^{\mathrm{C}}$ Chi-squared test, ${ }^{\mathrm{d}}$ Fisher's exact test, ${ }^{\mathrm{e}} \mathrm{Holm}$-Bonferroni. 
Table 3B. Univariate analyses comparing sociodemographic and clinical variables in pain classes for worst pain.

\begin{tabular}{|c|c|c|c|c|c|}
\hline & $\begin{array}{l}\text { No pain } \\
(n=21)\end{array}$ & $\begin{array}{l}\text { Class } 1 \\
(n=196)\end{array}$ & $\begin{array}{l}\text { Class } 2 \\
(n=47)\end{array}$ & $P$ & $\begin{array}{l}\text { Adjusted } \\
P^{e}\end{array}$ \\
\hline Age, years, mean $( \pm$ SD) & $70.7(6.5)$ & $65.7(8.4)$ & $64.9(9.0)$ & $0^{0.008}{ }^{a}$ & 0.159 \\
\hline BMI, mean $( \pm$ SD) & $24.8(4.3)$ & $25.7(4.7)$ & $27.1(4.7)$ & $0.112^{\mathrm{a}}$ & $1.000^{\mathrm{a}}$ \\
\hline $\begin{array}{l}\text { SCQ-19 total, median (Q1- } \\
\text { Q3) }\end{array}$ & $2.0(1.0-2.2)$ & $2(1.0-3.0)$ & $3.0(2.0-4.0)$ & $\mathbf{0 . 0 0 2}^{\mathrm{b}}$ & 0.049 \\
\hline $\begin{array}{l}\text { Pack-years smoking, median } \\
\text { (Q1-Q3) }\end{array}$ & $20.0(13.9-34.4)$ & $28.0(17.6-40.7)$ & $35.0(20.0-45.2)$ & $0.292^{\mathrm{b}}$ & 1.000 \\
\hline $\begin{array}{l}\text { Days with chest-tube, median } \\
\text { (Q1-Q3) }\end{array}$ & $2(1-4)$ & $3(2-5)$ & $3(2-5)$ & $0.383^{\mathrm{b}}$ & 1.000 \\
\hline Gender, $n(\%)$ & & & & $0.676^{\mathrm{c}}$ & 1.000 \\
\hline Female & $7(33.3)$ & $85(43.4)$ & $20(42.6)$ & & \\
\hline Cancer type, $n(\%)$ & & & & $0.248^{c}$ & 1.000 \\
\hline Adenocarcinoma & $9(42.9)$ & $115(58.7)$ & $25(53.2)$ & & \\
\hline Squamous cell & $11(52.4)$ & $47(27.8)$ & $23(31.1)$ & & \\
\hline
\end{tabular}




\begin{tabular}{|c|c|c|c|c|c|}
\hline Other & $1(4.8)$ & $25(14.8)$ & $8(10.8)$ & & \\
\hline Psychotropic medicine, $n(\%)$ & & & & $0.322^{c}$ & 1.000 \\
\hline Yes & $2(9.5)$ & 39 (19.9) & $12(25.5)$ & & \\
\hline Any pain medicine, $n(\%)$ & & & & $<0.001^{\mathrm{c}}$ & 0.001 \\
\hline Yes & $1(4.8)$ & $23(11.7)$ & $18(38.3)$ & & \\
\hline TNM stage of cancer, $n(\%)$ & & & & $0.847^{\mathrm{c}}$ & 1.000 \\
\hline IA & $4(21.1)$ & $62(34.1)$ & $14(31.8)$ & & \\
\hline IB & $8(42.1)$ & $57(31.3)$ & $14(31.8)$ & & \\
\hline II & $5(26.3)$ & $32(17.6)$ & $8(18.2)$ & & \\
\hline IIIA/IIIB & $2(10.5)$ & $31(17.0)$ & $8(18.2)$ & & \\
\hline Smoking group, $n(\%)$ & & & & $0.059^{\mathrm{d}}$ & 0.939 \\
\hline Smoked until surgery & $6(28.6)$ & $85(43.4)$ & $29(63.0)$ & & \\
\hline Stopped 1 year before surger & $14(66.7)$ & $97(49.5)$ & $15(32.6)$ & & \\
\hline Never smoked & $1(4.8)$ & $14(7.1)$ & $2(4.3)$ & & \\
\hline Surgery type, $n(\%)$ & & & & $<0.001^{\mathrm{d}}$ & $<0.005$ \\
\hline Lobectomy & $5(23.8)$ & $143(73.3)$ & $35(74.5)$ & & \\
\hline Bilobectomy & $2(9.5)$ & $15(7.7)$ & $2(4.3)$ & & \\
\hline
\end{tabular}




\begin{tabular}{|c|c|c|c|c|c|}
\hline Pulmectomy & $4(19.0)$ & $14(7.2)$ & $5(10.6)$ & & \\
\hline Wedge resection & $7(33.3)$ & $15(7.7)$ & $2(4.3)$ & & \\
\hline Thoracoscopic & $3(14.3)$ & $8(4.1)$ & $3(6.4)$ & & \\
\hline Additional surgery, $n(\%)$ & & & & $0.490^{c}$ & 1.000 \\
\hline Yes & $1(4.8)$ & $30(15.3)$ & $6(12.8)$ & & \\
\hline
\end{tabular}

BMI, body mass index; SCQ-19, Self-administered Comorbidity Questionnaire-19; Q1, lower quartile (25 ${ }^{\text {th }}$ percentile); Q3, upper quartile $\left(75^{\text {th }}\right.$ percentile). Classes identified by latent class mixed model $($ LCMM); No pain class; Class $1=$ elevated pain; Class $2=$ stable pain. ${ }^{\mathrm{a}} \mathrm{ANOVA},{ }^{\mathrm{b}} \mathrm{Kruskal}-\mathrm{Wallis},{ }^{\mathrm{c}} \mathrm{Chi}$-squared test, ${ }^{\mathrm{d}}$ Fisher's exact test, ${ }^{\mathrm{e}} \mathrm{Holm}$-Bonferroni. 


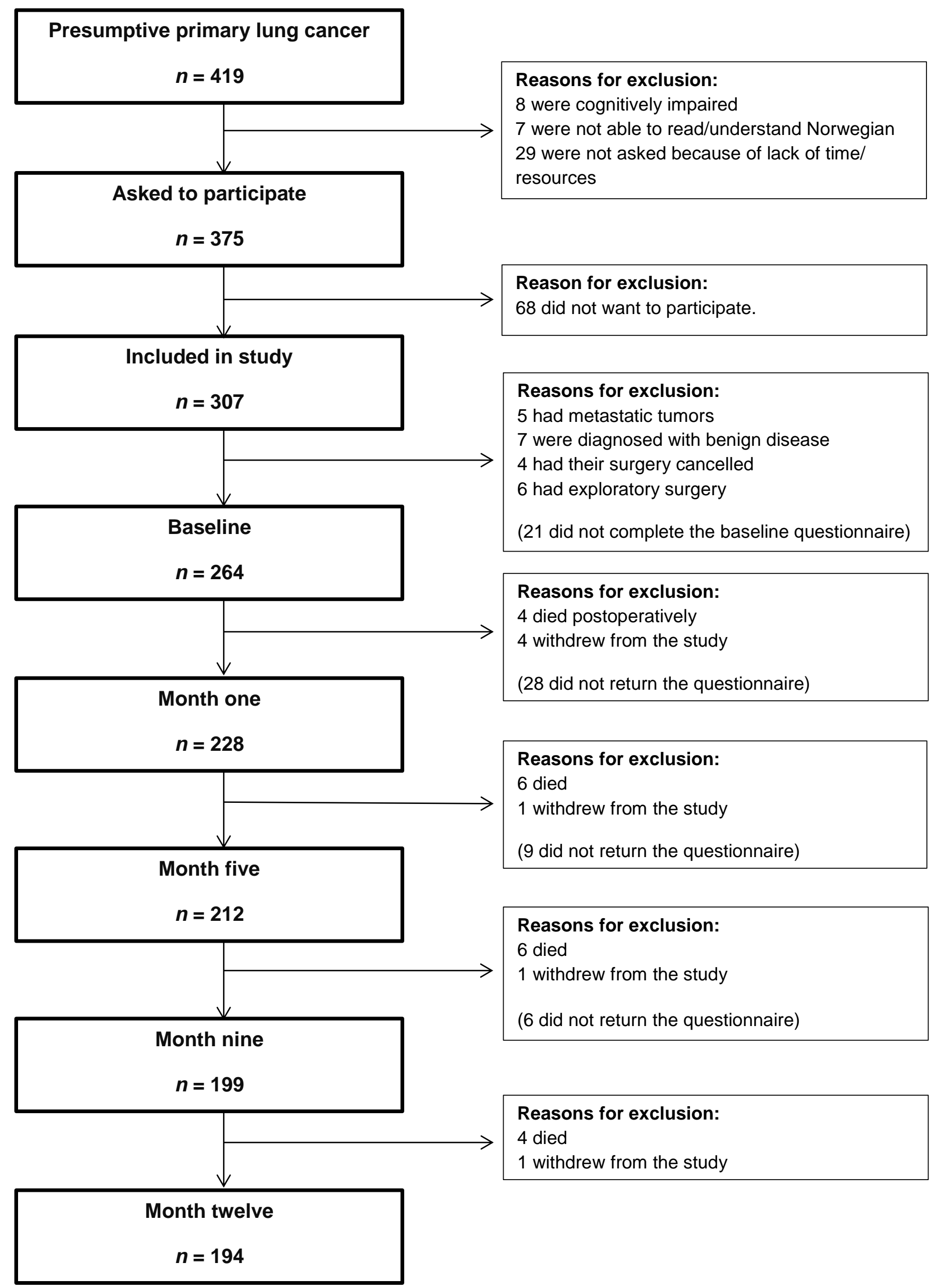




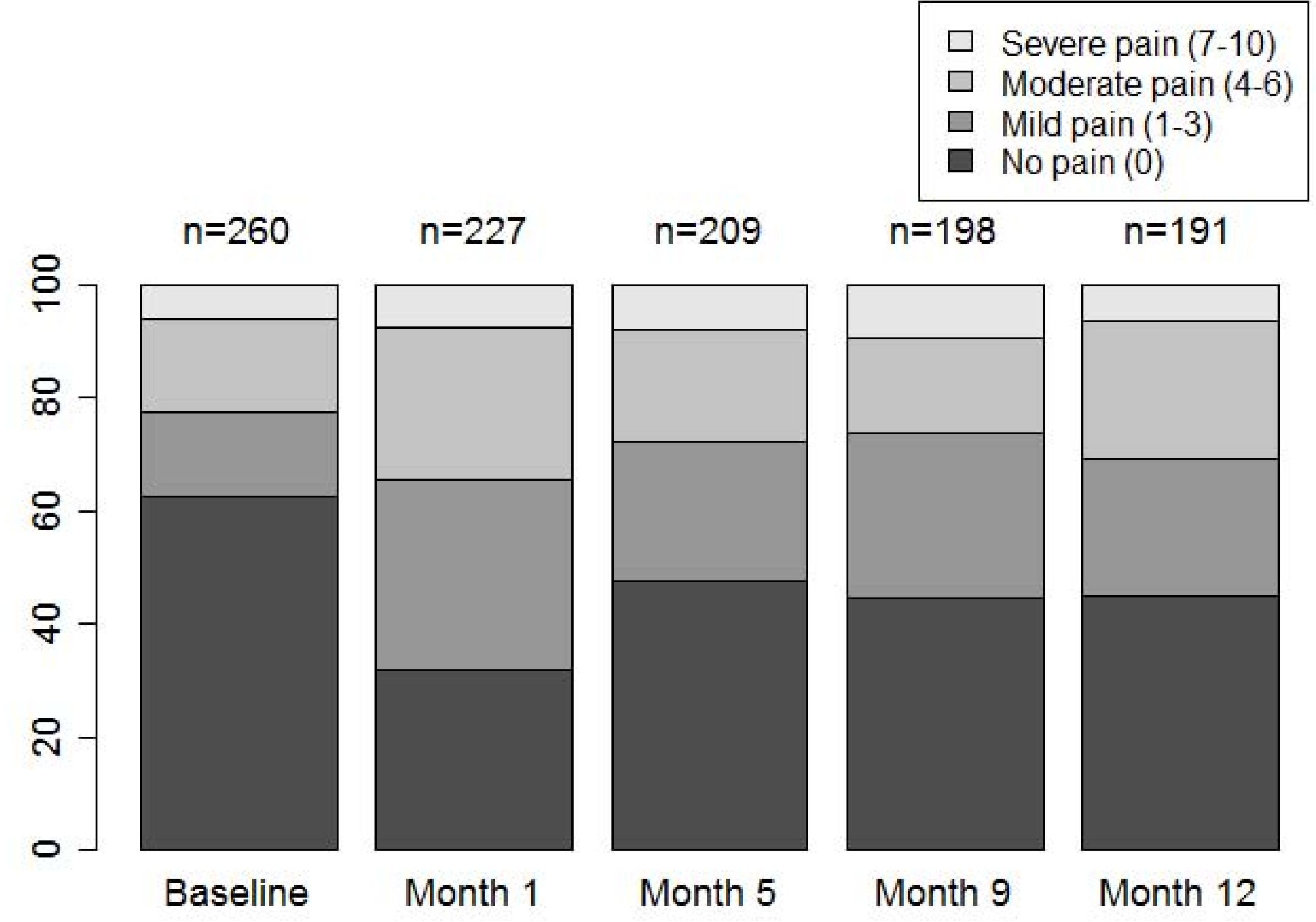




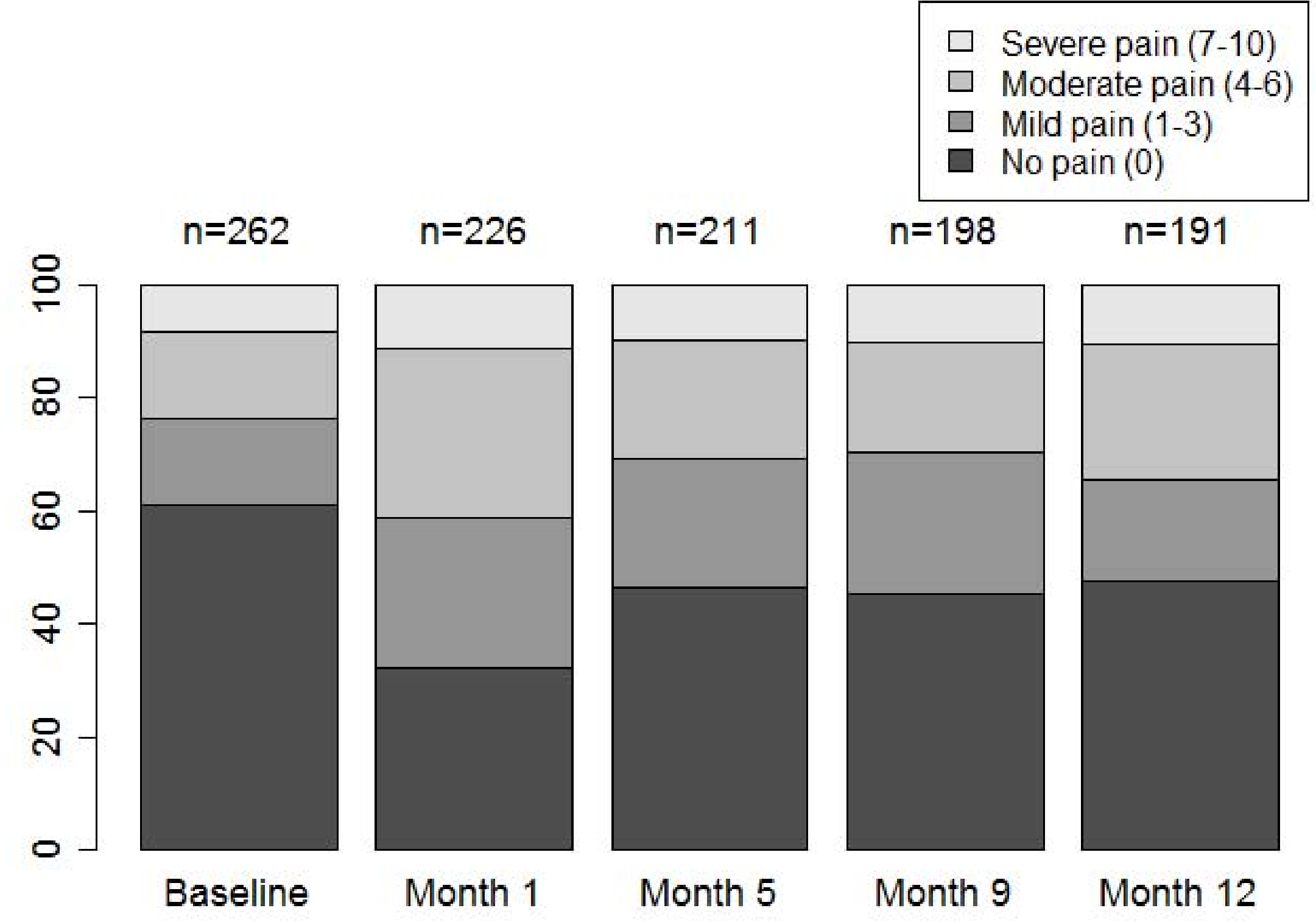




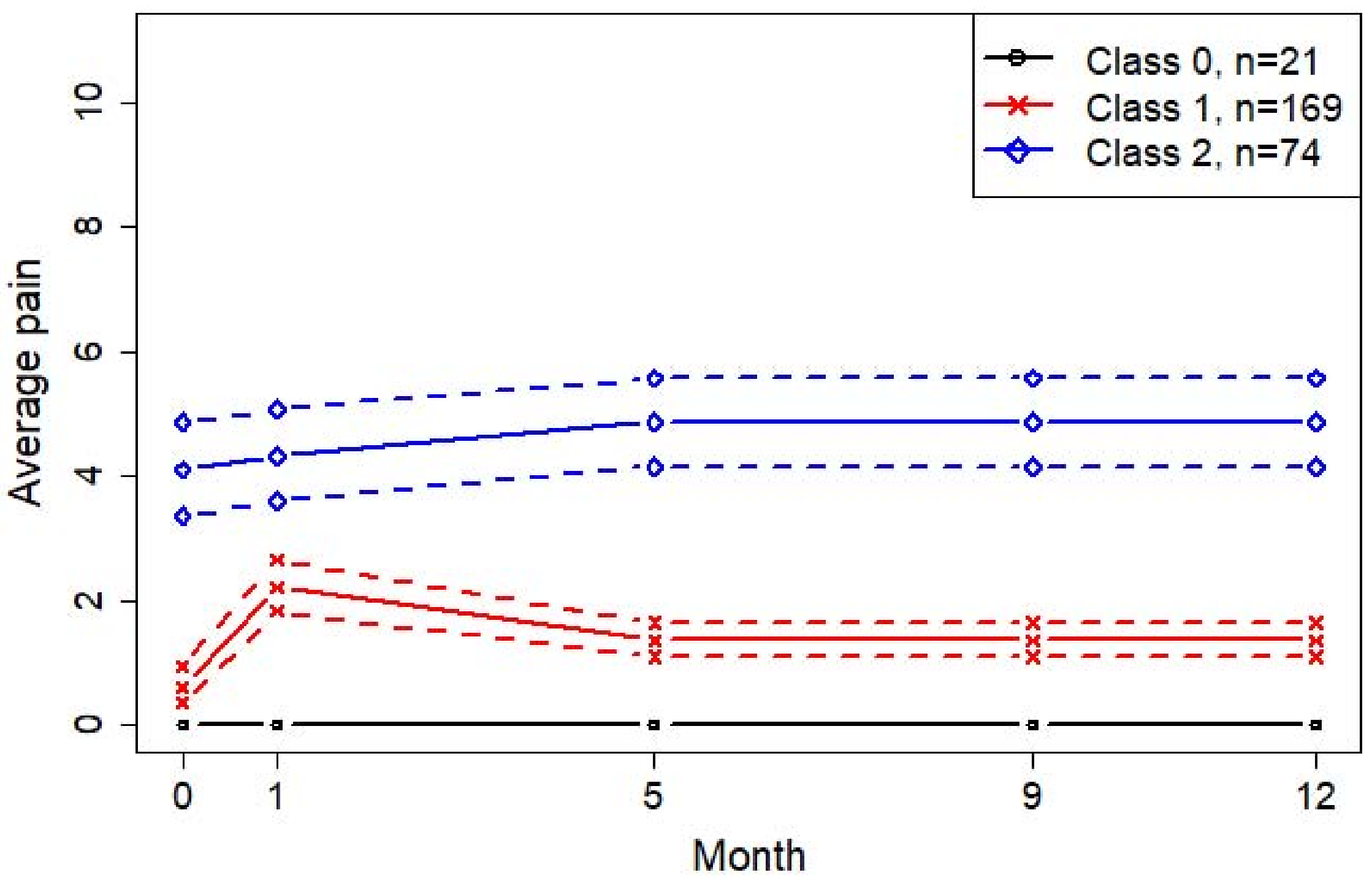




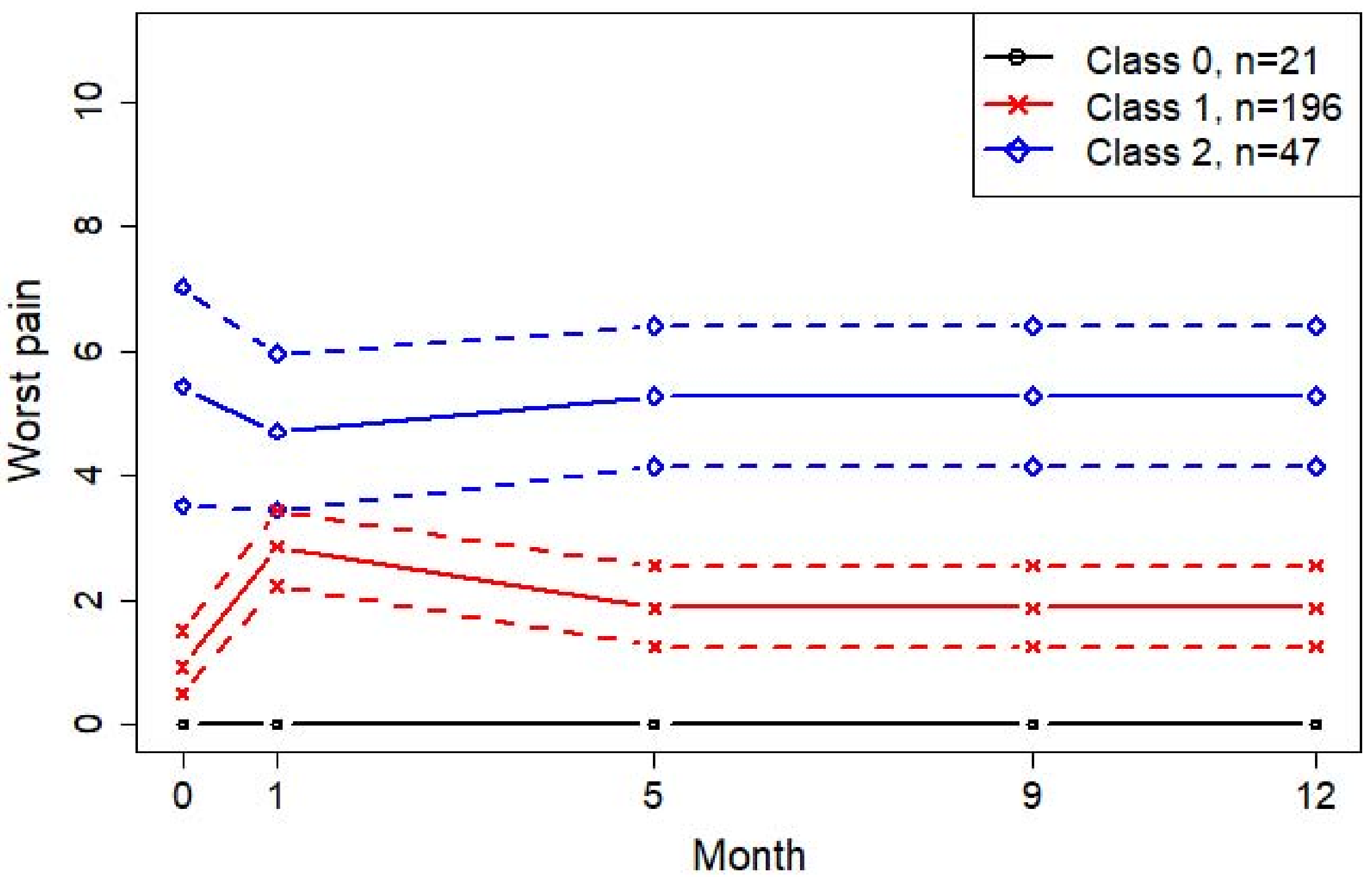


Supplementary Table S1. Location of pain during the patient trajectory reported on the body map in the Brief Pain Inventory.

\begin{tabular}{|c|c|c|c|c|c|}
\hline & Baseline & 1 month & 5 months & 9 months & 12 months \\
\hline & $N=264$ & $N=228$ & $N=212$ & $N=199$ & $N=194$ \\
\hline Location & $n(\%)$ & $n(\%)$ & $n(\%)$ & $n(\%)$ & $n(\%)$ \\
\hline Head/face/jaw & $21(8.0)$ & $17(7.5)$ & $12(5.7)$ & $11(5.2)$ & $15(7.7)$ \\
\hline Breast & $30(11.4)$ & 99 (43.4) & $53(25.0)$ & $41(20.6)$ & $50(25.8)$ \\
\hline Abdomen/stomach/pelvis & $17(6.5)$ & $28(12.3)$ & $25(11.8)$ & $18(9.0)$ & $18(9.3)$ \\
\hline Lower arms/hands & $27(10.2)$ & $18(8.0)$ & $21(9.9)$ & $12(6.0)$ & $13(6.7)$ \\
\hline Knees & $24(9.1)$ & $12(5.3)$ & $16(7.5)$ & $13(6.5)$ & $18(9.3)$ \\
\hline Hips & $35(13.3)$ & $21(9.2)$ & $27(12.7)$ & 35 (17.6) & $30(15.5)$ \\
\hline Neck/shoulders/upper arms & $52(19.7)$ & $81(35.5)$ & $59(27.8)$ & $53(26.7)$ & $61(31.4)$ \\
\hline Back pain & 57 (21.6) & 72 (31.6) & $58(27.4)$ & $58(29.1)$ & $50(25.8)$ \\
\hline
\end{tabular}




\begin{tabular}{|c|c|c|c|c|c|}
\hline Upper back & $22(8.3)$ & $53(23.2)$ & $19(9.0)$ & $22(11.1)$ & $22(11.3)$ \\
\hline Lower back & 48 (18.2) & $36(15.8)$ & $48(22.6)$ & $48(24.1)$ & $41(21.1)$ \\
\hline Legs/thighs/feet & $29(11.0)$ & $22(9.6)$ & $31(14.6)$ & $24(12.1)$ & $29(14.9)$ \\
\hline Throat & $2(0.8)$ & $3(1.3)$ & $7(3.3)$ & $7(3.5)$ & $2(1.0)$ \\
\hline
\end{tabular}

